\title{
The Japanese food score and risk of all-cause, CVD and cancer mortality: the Japan Collaborative Cohort Study
}

\author{
Emiko Okada $^{1}$, Koshi Nakamura ${ }^{2}$, Shigekazu Ukawa ${ }^{2}$, Kenji Wakai ${ }^{3}$, Chigusa Date ${ }^{4}$, Hiroyasu Iso ${ }^{5}$ and \\ Akiko Tamakoshi ${ }^{2 *}$ \\ ${ }^{1}$ Department of Nutritional Epidemiology and Shokuiku, National Institutes of Biomedical Innovation, Health and Nutrition, \\ 1-23-1 Toyama, Shinjuku-ku, Tokyo 162-8636, Japan \\ ${ }^{2}$ Department of Public Health, Hokkaido University Faculty of Medicine, North 15 West 7 Kita-ku, Sapporo 060-8638, Japan \\ ${ }^{3}$ Department of Preventive Medicine, Nagoya University Graduate School of Medicine, 65 Tsurumai-cho Showa-ku, Nagoya, \\ Aichi 466-8550, Japan \\ ${ }^{4}$ Department of Food Science and Nutrition, School of Human Science and Environment, University of Hyogo, 1-1-12 \\ Shinzaike-Honcho, Himeji, Hyogo 670-0092, Japan \\ ${ }^{5}$ Public Health, Department of Social and Environmental Medicine, Graduate School of Medicine, Osaka University, 2-2 \\ Yamadaoka, Suita, Osaka 565-0871, Japan
}

(Submitted 3 October 2017 - Final revision received 12 March 2018 - Accepted 10 May 2018 - First published online 20 June 2018 )

\section{Abstract}

Few studies have reported the association between the Japanese diet as food score and mortality. This study aimed to investigate adherence to the Japanese food score associated with all-cause, CVD and cancer mortality. A total of 58767 (23162 men and 34232 women) Japanese participants aged 40-79 years, who enrolled in the Japan Collaborative Cohort Study between 1988 and 1990, were included. The Japanese food score was derived from the components of seven food groups (beans and bean products, fresh fishes, vegetables, Japanese pickles, fungi, seaweeds and fruits) based on the FFQ. The total score ranged from 0 to 7 , and participants were divided into five categories based on scores (0-2, 3, 4, 5 and 6-7). Hazard ratios (HR) and 95\% CI for all-cause, CVD and cancer mortality based on sex were estimated using Cox proportional models. During the follow-up period until 2009, 11692 participants with all-cause, 3408 with CVD and 4247 with cancer died. The multivariable HR in the 6-7 and 0-2 Japanese food score groups were $0.93(95 \%$ CI $0.86,1.01)$ in men and 0.82 (95\% CI $0.75,0.90)$ in women for all-cause mortality and 0.89 (95\% CI $0.76,1.04)$ in men and 0.66 (95\% CI $0.56,0.77)$ in women for CVD mortality. Our findings suggest that adherence to the Japanese food score consisting of food combinations characterised by a Japanese diet may help in preventing all-cause and CVD mortality, especially in women.

\section{Key words: Diets: Scores: Japanese: Mortality: Cohort studies}

Recently, the associations between dietary patterns and overall diet and health outcomes are widely reported ${ }^{(1-6)}$. Dietary patterns are identified from a priori (via theoretical score or index) or a posteriori (via principal component analysis or factor analysis using food/nutritional data) ${ }^{(7)}$. However, a posteriori-defined dietary patterns can potentially differ for each study due to different population; dietary assessment methods, such as FFQ, dietary record and 24-h dietary recall; and analysis approach, such as consolidation of food items into food groups, number of factors to extract and labelling of factor components. The Mediterranean diet score, Healthy Eating Index (HEI), Alternate Healthy Eating Index (AHEI) and Dietary Approaches to Stop Hypertension (DASH) score are known as typical a priori dietary patterns worldwide. Higher adherence to the Mediterranean diet score, HEI, AHEI and DASH score was associated with lower rate of all-cause, CVD and cancer mortality $^{(8,9)}$.

The Japanese diet consists of a wide variety of foods ${ }^{(10)}$ and is different from diets in Western countries. Previous studies showed that intake of individual food groups such as fruits, vegetables, beans and fishes were inversely associated with all-cause and/or CVD mortality in Japan ${ }^{(11-13)}$. The score assessing the interactions between different dietary beneficial components of the Japanese diet is necessary to determine dietary patterns. Some studies reported that scores consisting of Japanese diet intake, that is a priori-defined Japanese diet score, decreased the risk of non-communicable diseases ${ }^{(14)}$ and functional disabilities ${ }^{(15)}$ among Japanese individuals. However, only a few a priori score or index evaluated the Japanese diet, and their effects on health are scarcely evident. 
In this study, the Japanese food score consisting of combined foods characterised by the Japanese diet was identified based on the previous studies using the data from the Japan Collaborative Cohort (JACC) Study. We investigated whether adherence to the Japanese food score is associated with the risk reduction of all-cause, CVD and cancer mortality among Japanese populations.

\section{Methods \\ Study population}

Between 1988 and 1990, participants were enrolled to participate in the JACC Study and were evaluated for cancer risk by assessing the impact of lifestyle factors on their health. Details of the study design have been described elsewhere ${ }^{(16)}$. In brief 110585 (46395 men and 64190 women) participants aged 40-79 years from forty-five regions throughout Japan were enrolled. Most participants were recruited during a health check-up by completing a self-administered questionnaire. Among the 110585 participants, those with a history of cancer ( $n$ 1461), stroke ( $n$ 1440) or myocardial infarction $(n=2774)$ at baseline were excluded. In addition, participants who lived in eleven study regions where the self-administered FFQ survey was not conducted at baseline ( $n$ 23 187) and those who did not complete the dietary survey ( $n$ 22941) were also excluded. Moreover, those with missing information on four or more items in the following thirteen food items constituting seven food group items in the FFQ ( $n$ 10) were also excluded: beans and beans products (boiled beans and tofu), fresh fishes, vegetables (spinach or garland chrysanthemum, carrots or pumpkin, tomatoes, cabbage or head lettuce and Chinese cabbage), Japanese pickles, fungi, seaweeds and fruits (citrus fruit and others). A total of 58767 participants were included in the analysis for this study. The study design was approved by the Ethical Review Board of Nagoya University School of Medicine.

\section{Dietary assessment and the Japanese food score}

Information on the intake of thirty-nine food items was assessed from a previously validated self-administered questionnaire, including an FFQ at baseline ${ }^{(17)}$. Most food items were assessed based on five frequency categories: never or seldom, 1-2 times/ month, 1-2 times/week, 3-4 times/week and almost daily. Rice and miso soup intakes were assessed using the number of bowls consumed daily. Beverages were assessed based on five frequency categories: almost never, 1-2 cups/month, 1-2 cups/ week, 3-4 cups/week and almost daily for green tea, tea, oolong tea and coffee. The frequency of alcohol consumption was assessed using the following categories: never, former, and current drinker ( $<1$ time/week, 1-2 times/week, 3-4 times/ week and almost daily). The consumption of each food was calculated by multiplying the frequency of consumption of each food with the portion size ${ }^{(18)}$, estimated from the validation study conducted $^{(17)}$. The average daily intake of nutrients was calculated by multiplying the frequency of consumption of each item by its nutrient content per serving and totalling the nutrient intake for all food items. Total energy and nutrient intakes were estimated based on the fifth edition of the Japan Food Table ${ }^{(19)}$, and nutrient intakes from food groups were adjusted based on the total energy intake using density methods.

The Japanese food score in this study was proposed based on several previous studies reporting dietary patterns as the Japanese diet using the principal components/factor analysis ${ }^{(20-23)}$. The Japanese food score consisted of seven food groups, namely, beans and beans products (boiled beans and tofu), fresh fish, vegetables (spinach or garland chrysanthemum, carrots or pumpkin, tomatoes, cabbage or head lettuce and Chinese cabbage), Japanese pickles, fungi, seaweeds and fruits (citrus fruit and others). Japanese individuals traditionally eat rice as staple foods and miso soup as soup stock with main and side dishes. We focused on the main and side dishes other than staple foods and soup stocks and assessed their Japanese food scores because $71.9 \%$ of the participants ate $\geq 3$ cups of rice, and $72.2 \%$ took miso soup every day in this study. For participants who left some questions with blanks/no answer on three or less items in the thirteen food items constituting the seven food group items, missing data were replaced with the median values based on their sex and geographical region. Because the most common frequency of food intake for many items is 1-2 times/week or 3-4 times/week in the present study, $\geq 3-4$ times/week was considered as the cut-off point ( 1 point is given) in participants who ate any food item from the seven food groups. Thus, the total Japanese food scores ranged from 0 to 7 , with higher scores indicating more Japanese food intake. Participants were divided into five categories based on the Japanese food scores for the analysis $(0-2,3,4,5$ or $6-7)$.

\section{Covariables}

Other information on demographic and lifestyle factors were collected using a self-administered questionnaire at baseline: educational levels, occupation, marital status, number of children, medical history (such as cancer, stroke, myocardial infarction, diabetes and hypertension), family history, health check-up status, smoking status, environmental tobacco smoke, alcohol drinking status, sports habits, sleep duration, height, weight, weight at 20 years old, blood pressure, number of pregnancy and parity (for women) and menarche and menopausal age (for women). BMI was calculated as weight $(\mathrm{kg})$ divided by height $\left(\mathrm{m}^{2}\right)$.

\section{Follow-up}

Mortality data were centralised at the Ministry of Health and Welfare, and the underlying causes of deaths were coded using National Vital Statistics codes in accordance with the 10th revision of the International Classification of Disease-10. Follow-up was completed at the end of 2009 in most regions but was terminated at the end of 1999 in four regions, 2003 in another four regions and 2008 in two regions. The censoring was determined when follow-period was completed from study enrolment. Participants who moved from their regions during the study period were treated as censored at the time of their move. Cause-specific mortality was individually coded for cancer (C02-97) and CVD (I05-99). 


\section{Statistical analysis}

Sex-specific age-adjusted and multivariate hazard ratios (HR) and $95 \% \mathrm{CI}$ for the mortality risk related to all-cause, cancer and CVD were evaluated based on the Japanese food scores of 3 to 6-7 and compared with those of $0-2$ using the Cox proportional hazard model. The linear relationship was assessed using the Japanese food scores as continuous variables, and $P_{\text {trend }}$ values were obtained. The following variables were included in the multivariate models as potential confounders: age (continuous variable), geographical region, BMI $(<18 \cdot 5,18 \cdot 5-24 \cdot 9,25 \cdot 0$ $29 \cdot 9, \geq 30 \cdot 0 \mathrm{~kg} / \mathrm{m}^{2}$ or unknown), educational levels $(<13, \geq 13$ years or unknown), smoking status (never, former, current smoker or unknown), alcohol drinking status (never, former, current drinker or unknown), sports habits (rarely, 1-2, 3-4, $\geq 5 \mathrm{~h}$ /week or unknown), sleep duration (<6.0, 6.0-6.9, 7.0-7.9, $8 \cdot 0-8 \cdot 9, \geq 9$ h or unknown), history of hypertension and diabetes (yes, no, or unknown), and total daily energy intake $(<5895,5896-7242,7243-8745$ or $\geq 8746 \mathrm{~kJ} / \mathrm{d}(<1409$, 1410 $1731,1732-2090$ or $\geq 2091 \mathrm{kcal} / \mathrm{d})$ in men and $<4971,4972$ $5858,5869-6853$ or $6854 \mathrm{~kJ} / \mathrm{d}(<1188,1189-1400,1401-1638$ or $\geq 1639 \mathrm{kcal} / \mathrm{d}$ ) in women). In addition, we analysed the data of women using a different category (0-3, 4, 5, 6 and 7) because the number of women with high Japanese food scores was higher than of men with high Japanese food scores in the present study. The SAS statistical package for Windows (version 9.4; SAS Institute Inc.) was used to perform all statistical analyses. Differences were considered statistically significant at $P<0.05$.

\section{Results}

Tables 1 (men) and 2 (women) show the demographic and nutritional baseline characteristics of the participants according to the adherence to Japanese food scores based on sex. Male participants with higher Japanese food scores were older, more educated, had longer duration of sleep and sports hours, and were not current smokers, whereas female participants with higher Japanese food scores were older, had higher educational levels, had longer sports hours, and were not current smokers, compared with participants with lower Japanese food scores. Those with higher adherence to the Japanese food score had a higher mean intake of almost all nutrients but had lower carbohydrate (\% of energy) and the Na:K ratio (in both men and women).

During the 373232 person-years and a median follow-up period of 18.9 years, 6309, 1674 and 2490 deaths were attributed to all-cause, CVD and cancer mortality in men, respectively. In women, during the 594634 person-years and a median follow-up period of $19 \cdot 4$ years, 5383, 1734, 1757 deaths were attributed to all-cause, CVD and cancer mortality, respectively. Tables 3 (men) and 4 (women) show the Cox proportional hazard model results between the adherence to the Japanese food score and all-cause, CVD, and cancer mortality based on sex. In men, higher Japanese food score was associated with a marginally decreased risk of all-cause mortality, with multivariate HR of 0.96 (95\% CI $0.88,1.04), 0.92$ (95\% CI 0.84, 1.00), 0.95 (95\% CI 0.88, 1.03) and 0.93 (95\% CI
$0 \cdot 86,1.01)$ for scores $3,4,5$ and $6-7$, respectively; $P_{\text {trend }}$ was 0.067. In women, higher Japanese food score was associated with decreased risk of all-cause mortality, with multivariate HR of 0.92 (95\% CI 0.82, 1.03), 0.99 (95\% CI 0.89, 1.09), 0.85 (95\% CI $0.77,0.94)$ and $0.82(95 \%$ CI $0.75,0.90)$ for scores $3,4,5$ and $6-7$, respectively; $P_{\text {trend }}$ was $<0 \cdot 001$. CVD mortality decreased when the Japanese food score was higher, with multivariate HR of 0.80 (95\% CI $0.67,0.97), 0.87$ (95\% CI 0.74, 1.03), 0.76 (95\% CI $0.64,0.89)$ and 0.66 (95\% CI 0.56, 0.77) for scores 3, 4, 5 and 6-7, respectively $\left(P_{\text {trend }}<0 \cdot 001\right)$. However, regarding cancer mortality, no significant associations were observed with the Japanese food score in both men and women. Using separate cut-off points for the Japanese food score in men (0-2, 3, 4, 5 and 6-7) and women (0-3, 4, 5, 6 and 7) yielded similar results (data not shown).

\section{Discussion}

The Japanese food score comprising of food groups characterised in the Japanese diet was identified and evaluated based on the data from Japanese individuals who participated in a large prospective cohort study. It was determined by adding the food intakes based on the Japanese diet, which is diverse $^{(10)}$ and commonly taken foods in a normal dietary habit. The score from the food intake frequency also provided an easily accessible public health information to the general population. In addition, adherence to the Japanese food score may help in preventing all-cause and CVD mortality, especially in women, but not in cancer mortality.

A previous Japanese study has reported the following reducedsalt Japanese food score: egg intake of $\leq 2$ eggs/week, $\geq 1$ fish intake in $2 \mathrm{~d}$, meat intake of $\leq 2$ times/week, $\geq 1$ Japanese pickle intake/d, infrequent intake of soup with noodles, use of low-salt soya sauce and occasional drinking ${ }^{(14)}$. Higher reduced-salt Japanese food score was associated with lower rate of all-cause and CVD mortality, but not on cancer mortality ${ }^{(14)}$. Another Japanese study reported the Japanese diet index score based on the consumption of a good (rice, miso soup, seaweeds, pickles, green and yellow vegetables, fish and green tea) and bad foods (beef, pork and coffee) ${ }^{(15)}$. The Japanese diet index score was associated with decreased risk of functional disability incidence among elderly people ${ }^{(15)}$. The components of the Japanese food score measured using the intake frequency of beans and bean products, fresh fishes, vegetables, Japanese pickles, fungi, seaweeds, and fruits in this study were different from those in previous studies ${ }^{(14,15)}$. However, our results were consistent with those of previous studies revealing the beneficial effects of the score in evaluating Japanese diet on all-cause mortality and health-related outcomes, but not on cancer mortality.

Some individual food groups (Japanese pickles, fruits, vegetables and fishes) were inversely associated with all-cause and/ or CVD mortality in previous studies ${ }^{(14,24,25)}$. Many Japanese pickles composing part of the Japanese food score are traditionally made using salt, and Japanese people mainly consume salt from foods (such as Japanese pickles) and seasonings (such as soya sauce and miso). In general, high salt intake is likely to increase the risk of stroke and total $\mathrm{CVD}^{(26)}$ through 
Table 1. Demographic and nutrient baseline characteristics of participants according to the Japanese food scores (men)

(Mean values and standard deviations; percentages)

\begin{tabular}{|c|c|c|c|c|c|c|c|c|c|c|}
\hline & \multicolumn{2}{|c|}{ Score 0-2 (n 4694) } & \multicolumn{2}{|c|}{ Score 3 ( $n$ 3502) } & \multicolumn{2}{|c|}{ Score 4 ( $n$ 4238) } & \multicolumn{2}{|c|}{ Score 5 ( $n$ 4752) } & \multicolumn{2}{|c|}{ Score 6-7 (n 5976) } \\
\hline & Mean & SD & Mean & SD & Mean & SD & Mean & SD & Mean & SD \\
\hline Age (years) & $54 \cdot 2$ & $10 \cdot 1$ & $55 \cdot 3$ & $10 \cdot 1$ & $55 \cdot 8$ & 9.9 & $56 \cdot 7$ & $9 \cdot 7$ & $57 \cdot 2$ & $9 \cdot 6$ \\
\hline \multicolumn{11}{|l|}{ Educational level (years) $(\%)^{\star}$} \\
\hline$<13$ & \multicolumn{2}{|c|}{$76 \cdot 2$} & \multicolumn{2}{|c|}{$76 \cdot 2$} & \multicolumn{2}{|c|}{$75 \cdot 1$} & \multicolumn{2}{|c|}{74.5} & \multicolumn{2}{|c|}{$72 \cdot 7$} \\
\hline$\geq 13$ & \multirow{2}{*}{\multicolumn{2}{|c|}{$16 \cdot 3$}} & \multicolumn{2}{|c|}{$17 \cdot 0$} & & & 16 & & & \\
\hline $\mathrm{BMI}\left(\mathrm{kg} / \mathrm{m}^{2}\right)(\%)^{\star}$ & & & & & & & & & & \\
\hline$<18.5$ & & & & & & & & & & \\
\hline $18 \cdot 5-24.9$ & & & & & & & & & & \\
\hline $25 \cdot 0-29 \cdot 9$ & & & & & & & 16 & & & \\
\hline$\geq 30.0$ & & & & & & & 0 & & & \\
\hline Smoking status (\%)* & & & & & & & & & & \\
\hline Current smoker & & & & & & & 51 & & & \\
\hline Former smoker & & & & & & & 24 & & & \\
\hline Never smoker & & & & & & & 21 & & & \\
\hline Alcohol drinking status (\%)* & & & & & & & & & & \\
\hline Current drinker & & & & & & & 74 & & & \\
\hline Former drinker & & & & & & & & & & \\
\hline Never drinker & & & & & & & 20 & & & \\
\hline Sport habits (h/week) $(\%)^{\star}$ & & & & & & & & & & \\
\hline Rarely & & & & & & & 66 & & & \\
\hline $1-2$ & & & & & & & 16 & & & \\
\hline $3-4$ & & & & & & & & & & \\
\hline$\geq 5$ & & & & & & & & & & \\
\hline Sleep duration $(\mathrm{h} / \mathrm{d})(\%)^{*}$ & & & & & & & & & & \\
\hline$<6$ & & & & & & & 2 & & & \\
\hline $6-<7$ & & & & & & & 13 & & & \\
\hline $7-<8$ & & & & & & & 33 & & & \\
\hline $8-<9$ & & & & & & & 37 & & & \\
\hline$\geq 9$ & & & & & & & 10 & & & \\
\hline Hypertension (\%)* & & & & & & & & & & \\
\hline Diabetes $(\%)^{*}$ & & & & & & & 10 & & & \\
\hline Dietary intake & & & & & & & 6 & & & \\
\hline Energy intake (kJ) & 6406 & 2000 & 6945 & 1908 & 7326 & 1962 & 7669 & 1992 & 8226 & 2100 \\
\hline Energy intake (kcal) & 1531 & 478 & 1660 & 456 & 1751 & 469 & 1833 & 476 & 1966 & 502 \\
\hline Protein (\% energy) & 11.2 & $2 \cdot 4$ & $12 \cdot 0$ & $2 \cdot 3$ & $12 \cdot 5$ & 2.4 & $13 \cdot 1$ & $2 \cdot 3$ & $13 \cdot 8$ & 2.3 \\
\hline Fat (\% energy) & 14.9 & $5 \cdot 1$ & $16 \cdot 1$ & 4.8 & $16 \cdot 6$ & 4.7 & 17.5 & 4.6 & $18 \cdot 4$ & 4.5 \\
\hline Carbohydrate (\% energy) & $59 \cdot 1$ & $11 \cdot 5$ & $58 \cdot 0$ & $10 \cdot 5$ & $57 \cdot 4$ & $10 \cdot 4$ & $57 \cdot 1$ & 9.5 & $56 \cdot 1$ & $8 \cdot 8$ \\
\hline $\mathrm{Na}(\mathrm{mg} / 4184 \mathrm{~kJ}(\mathrm{mg} / 1000 \mathrm{kcal}))$ & 1066 & 581 & 1156 & 525 & 1205 & 499 & 1265 & 473 & 1331 & 429 \\
\hline $\mathrm{K}(\mathrm{mg} / 4184 \mathrm{~kJ}(\mathrm{mg} / 1000 \mathrm{kcal}))$ & 1018 & 302 & 1145 & 307 & 1208 & 306 & 1291 & 312 & 1390 & 313 \\
\hline Na:K ratio $(\mathrm{mg} / \mathrm{mg})$ & 1.03 & 0.43 & 1.00 & 0.35 & 1.00 & 0.32 & 0.98 & 0.28 & 0.96 & 0.24 \\
\hline $\mathrm{Ca}(\mathrm{mg} / 4184 \mathrm{~kJ}(\mathrm{mg} / 1000 \mathrm{kcal}))$ & 231 & 97 & 255 & 93 & 265 & 90 & 281 & 88 & 294 & 82 \\
\hline $\mathrm{Mg}(\mathrm{mg} / 4184 \mathrm{~kJ}(\mathrm{mg} / 1000 \mathrm{kcal}))$ & 150 & 28 & 157 & 26 & 161 & 25 & 167 & 25 & 172 & 24 \\
\hline $\mathrm{Fe}(\mathrm{mg} / 4184 \mathrm{~kJ}(\mathrm{mg} / 1000 \mathrm{kcal}))$ & 3.75 & 1.28 & 4.09 & 1.23 & 4.29 & 1.17 & 4.54 & 1.17 & 4.80 & $1 \cdot 12$ \\
\hline $\begin{array}{l}\beta \text {-Carotene equivalents } \\
(\mu \mathrm{g} / 4184 \mathrm{~kJ}(\mu \mathrm{g} / 1000 \mathrm{kcal}))^{*}\end{array}$ & 114 & 68 & 142 & 81 & 158 & 86 & 180 & 91 & 205 & 88 \\
\hline $\begin{array}{l}\text { Retinol equivalents } \\
(\mu \mathrm{g} / 4184 \mathrm{~kJ}(\mu \mathrm{g} / 1000 \mathrm{kcal}))^{*}\end{array}$ & 362 & 424 & 394 & 410 & 424 & 436 & 453 & 433 & 497 & 432 \\
\hline Vitamin D $(\mu \mathrm{g} / 4184 \mathrm{~kJ}(\mu \mathrm{g} / 1000 \mathrm{kcal}))$ & $2 \cdot 72$ & 1.44 & 3.34 & 1.65 & 3.71 & 1.75 & $4 \cdot 14$ & 1.72 & 4.83 & 1.68 \\
\hline Vitamin $\mathrm{K}(\mu \mathrm{g} / 4184 \mathrm{~kJ}(\mu \mathrm{g} / 1000 \mathrm{kcal}))$ & $69 \cdot 1$ & $34 \cdot 3$ & $89 \cdot 3$ & 39.8 & $97 \cdot 3$ & $38 \cdot 8$ & $107 \cdot 1$ & $39 \cdot 1$ & $116 \cdot 5$ & $37 \cdot 2$ \\
\hline Vitamin $B_{1}(\mathrm{mg} / 4184 \mathrm{~kJ}(\mathrm{mg} / 1000 \mathrm{kcal}))$ & 0.52 & 0.09 & 0.55 & 0.09 & 0.57 & 0.09 & 0.59 & 0.08 & 0.61 & 0.08 \\
\hline Vitamin $B_{2}(\mathrm{mg} / 4184 \mathrm{~kJ}(\mathrm{mg} / 1000 \mathrm{kcal}))$ & 0.51 & 0.20 & 0.55 & 0.19 & 0.57 & 0.19 & 0.60 & 0.19 & 0.64 & 0.18 \\
\hline Niacin $(\mathrm{mg} / 4184 \mathrm{~kJ}(\mathrm{mg} / 1000 \mathrm{kcal}))$ & $10 \cdot 3$ & $1 \cdot 8$ & $10 \cdot 6$ & $1 \cdot 7$ & $10 \cdot 8$ & 1.7 & $11 \cdot 1$ & $1 \cdot 6$ & 11.5 & 1.6 \\
\hline Vitamin $B_{6}(\mathrm{mg} / 4184 \mathrm{~kJ}(\mathrm{mg} / 1000 \mathrm{kcal}))$ & 0.59 & 0.12 & 0.65 & 0.12 & 0.68 & 0.12 & 0.71 & 0.12 & 0.76 & 0.12 \\
\hline Vitamin $B_{12}(\mathrm{mg} / 4184 \mathrm{~kJ}(\mathrm{mg} / 1000 \mathrm{kcal}))$ & 3.36 & 1.96 & 3.73 & 1.92 & 4.01 & 1.98 & 4.33 & 1.97 & $4 \cdot 76$ & 1.90 \\
\hline Folate $(\mu \mathrm{g} / 4184 \mathrm{~kJ}(\mu \mathrm{g} / 1000 \mathrm{kcal}))$ & 149 & 61 & 170 & 63 & 181 & 63 & 194 & 64 & 209 & 63 \\
\hline Vitamin C (mg/4184 kJ (mg/1000 kcal)) & $41 \cdot 1$ & 18.9 & $51 \cdot 2$ & $21 \cdot 2$ & $55 \cdot 7$ & $21 \cdot 3$ & $60 \cdot 9$ & 21.5 & 67.4 & $21 \cdot 1$ \\
\hline SFA (\% energy) & $5 \cdot 26$ & $2 \cdot 28$ & 5.56 & $2 \cdot 10$ & 5.63 & 1.98 & 5.85 & 1.90 & 6.05 & 1.78 \\
\hline MUFA (\% energy) & $5 \cdot 23$ & 1.95 & 5.67 & 1.8 .9 & 5.85 & 1.84 & $6 \cdot 15$ & 1.80 & $6 \cdot 50$ & 1.78 \\
\hline PUFA (\% energy) & 3.92 & 1.30 & 4.25 & 1.27 & 4.47 & 1.24 & 4.72 & 1.22 & 4.99 & $1 \cdot 15$ \\
\hline$n-3$ PUFA (\% energy) & 0.68 & 0.27 & 0.79 & 0.29 & 0.86 & 0.30 & 0.94 & 0.30 & 1.04 & 0.28 \\
\hline$n-6$ PUFA (\% energy) & 3.23 & 1.09 & 3.44 & 1.06 & 3.59 & 1.02 & 3.76 & 1.00 & 3.93 & 0.93 \\
\hline Cholesterol (mg/4184 kJ (mg/1000 kcal)) & 114 & 54 & 127 & 52 & 133 & 51 & 142 & 49 & 148 & 45 \\
\hline Dietary fibre $(\mathrm{g} / 4184 \mathrm{~kJ}(\mathrm{~g} / 1000 \mathrm{kcal}))$ & $6 \cdot 18$ & 1.59 & 6.75 & 1.56 & $7 \cdot 10$ & 1.55 & 7.50 & 1.55 & 8.02 & 1.55 \\
\hline
\end{tabular}

* Unknown percentage was not shown. 
Table 2. Demographic and nutrient baseline characteristics of participants according to the Japanese food scores (women) (Mean values and standard deviations; percentages)

\begin{tabular}{|c|c|c|c|c|c|c|c|c|c|c|}
\hline & \multicolumn{2}{|c|}{ Score 0-2 (n 3766) } & \multicolumn{2}{|c|}{ Score 3 ( $n$ 4096) } & \multicolumn{2}{|c|}{ Score 4 (n 6291) } & \multicolumn{2}{|c|}{ Score 5 ( $n$ 8242) } & \multicolumn{2}{|c|}{ Score 6-7 $(n$ 13210) } \\
\hline & Mean & SD & Mean & SD & Mean & SD & Mean & SD & Mean & SD \\
\hline Age (years) & $56 \cdot 3$ & $10 \cdot 6$ & 55.9 & $10 \cdot 2$ & $55 \cdot 9$ & $10 \cdot 0$ & $56 \cdot 1$ & $9 \cdot 8$ & $56 \cdot 7$ & 9.5 \\
\hline \multicolumn{11}{|l|}{ Educational level (years) (\%)* } \\
\hline$<13$ & \multicolumn{2}{|c|}{$84 \cdot 2$} & \multicolumn{2}{|c|}{83.9} & \multirow{2}{*}{\multicolumn{2}{|c|}{$82 \cdot 8$}} & \multicolumn{2}{|c|}{81.6} & \multicolumn{2}{|c|}{$79 \cdot 6$} \\
\hline$\geq 13$ & \multicolumn{2}{|c|}{$7 \cdot 6$} & \multicolumn{2}{|c|}{8.5} & & & & & & \\
\hline $\mathrm{BMI}\left(\mathrm{kg} / \mathrm{m}^{2}\right)(\%)^{*}$ & & & & & & & & & & \\
\hline$<18.5$ & & & & & 5 & & & & & \\
\hline $18.5-24.9$ & & & & & 67 & & & & & \\
\hline $25 \cdot 0-29 \cdot 9$ & & & & & 20 & & & & & \\
\hline$\geq 30.0$ & & & & & 2 & & & & & \\
\hline Smoking status (\%)* & & & & & & & & & & \\
\hline Current smoker & & & & & 5 & & & & & \\
\hline Former smoker & & & & & 1 & & & & & \\
\hline Never smoker & & & & & 86 & & & & & \\
\hline Alcohol drinking status (\%)* & & & & & & & & & & \\
\hline Current drinker & & & & & 23 & & & & & \\
\hline Former drinker & & & & & 1 & & & & & \\
\hline Never drinker & & & & & 71 & & & & & \\
\hline Sports habits (h/week) $(\%)^{*}$ & & & & & & & & & & \\
\hline Rarely & & & & & 75 & & & & & \\
\hline $1-2$ & & & & & 12 & & & & & \\
\hline $3-4$ & & & & & 5 & & & & & \\
\hline$\geq 5$ & & & & & 3 & & & & & \\
\hline Sleep duration $(\mathrm{h} / \mathrm{d})(\%)^{*}$ & & & & & & & & & & \\
\hline$<6$ & & & & & 5 & & & & & \\
\hline $6-<7$ & & & & & 21 & & & & & \\
\hline $7-<8$ & & & & & 37 & & & & & \\
\hline $8-<9$ & & & & & 25 & & & & & \\
\hline$\geq 9$ & & & & & 5 & & & & & \\
\hline Hypertension (\%)* & & & & & & & & & & \\
\hline Diabetes $(\%)^{*}$ & & & & & 20 & & & & & \\
\hline Dietary intake & & & & & 3 & & & & & \\
\hline Energy intake (kJ) & 4983 & 1502 & 5452 & 1439 & 5757 & 1456 & 6025 & 1414 & 6489 & 1481 \\
\hline Energy intake (kcal) & 1191 & 359 & 1303 & 344 & 1376 & 348 & 1440 & 338 & 1551 & 354 \\
\hline Protein (\% energy) & 13.0 & $2 \cdot 3$ & 13.7 & $2 \cdot 2$ & $14 \cdot 3$ & $2 \cdot 2$ & 14.9 & $2 \cdot 2$ & $15 \cdot 8$ & 2.1 \\
\hline Fat (\% energy) & 17.8 & 5.4 & 19.1 & $5 \cdot 0$ & $19 \cdot 9$ & 4.8 & $20 \cdot 7$ & 4.5 & $21 \cdot 8$ & $4 \cdot 3$ \\
\hline Carbohydrate (\% energy) & $66 \cdot 4$ & 8.2 & $65 \cdot 0$ & $7 \cdot 3$ & 63.7 & $7 \cdot 0$ & $62 \cdot 7$ & 6.5 & $60 \cdot 9$ & $6 \cdot 1$ \\
\hline $\mathrm{Na}(\mathrm{mg} / 4184 \mathrm{~kJ}(\mathrm{mg} / 1000 \mathrm{kcal}))$ & 1188 & 641 & 1259 & 580 & 1329 & 532 & 1400 & 495 & 1467 & 444 \\
\hline $\mathrm{K}(\mathrm{mg} / 4184 \mathrm{~kJ}(\mathrm{mg} / 1000 \mathrm{kcal}))$ & 1236 & 320 & 1376 & 312 & 1466 & 311 & 1554 & 309 & 1662 & 300 \\
\hline $\mathrm{Na}: \mathrm{K}$ ratio $(\mathrm{mg} / \mathrm{mg})$ & 0.95 & 0.42 & 0.91 & 0.34 & 0.90 & 0.31 & 0.90 & 0.27 & 0.88 & 0.23 \\
\hline $\mathrm{Ca}(\mathrm{mg} / 4184 \mathrm{~kJ}(\mathrm{mg} / 1000 \mathrm{kcal}))$ & 275 & 104 & 302 & 97 & 321 & 96 & 338 & 91 & 356 & 85 \\
\hline $\mathrm{Mg}(\mathrm{mg} / 4184 \mathrm{~kJ}(\mathrm{mg} / 1000 \mathrm{kcal}))$ & 171 & 24 & 177 & 23 & 182 & 22 & 188 & 22 & 193 & 21 \\
\hline $\mathrm{Fe}(\mathrm{mg} / 4184 \mathrm{~kJ}(\mathrm{mg} / 1000 \mathrm{kcal}))$ & 4.33 & 1.39 & 4.62 & 1.29 & 4.88 & 1.23 & $5 \cdot 16$ & $1 \cdot 20$ & 5.45 & 1.12 \\
\hline $\begin{array}{l}\beta \text {-Carotene equivalents } \\
(\mu \mathrm{g} / 4184 \mathrm{~kJ}(\mu \mathrm{g} / 1000 \mathrm{kcal}))^{\star}\end{array}$ & 154 & 92 & 190 & 105 & 211 & 107 & 238 & 110 & 262 & 101 \\
\hline $\begin{array}{l}\text { Retinol equivalents } \\
(\mu \mathrm{g} / 4184 \mathrm{~kJ}(\mu \mathrm{g} / 1000 \mathrm{kcal}))^{\star}\end{array}$ & 425 & 536 & 465 & 533 & 489 & 504 & 514 & 497 & 563 & 497 \\
\hline Vitamin $D(\mu \mathrm{g} / 4184 \mathrm{~kJ}(\mu \mathrm{g} / 1000 \mathrm{kcal}))$ & $3 \cdot 21$ & 1.59 & 3.80 & $1 \cdot 86$ & 4.25 & 1.96 & 4.78 & 1.98 & 5.73 & 1.88 \\
\hline Vitamin $\mathrm{K}(\mu \mathrm{g} / 4184 \mathrm{~kJ}(\mu \mathrm{g} / 1000 \mathrm{kcal}))$ & $86 \cdot 9$ & $41 \cdot 7$ & $107 \cdot 1$ & $44 \cdot 1$ & $118 \cdot 3$ & $45 \cdot 8$ & $129 \cdot 3$ & $44 \cdot 2$ & $139 \cdot 2$ & 40.0 \\
\hline Vitamin $B_{1}(\mathrm{mg} / 4184 \mathrm{~kJ}(\mathrm{mg} / 1000 \mathrm{kcal}))$ & 0.61 & 0.07 & 0.64 & 0.07 & 0.65 & 0.07 & 0.67 & 0.07 & 0.69 & 0.07 \\
\hline Vitamin $B_{2}(\mathrm{mg} / 4184 \mathrm{~kJ}(\mathrm{mg} / 1000 \mathrm{kcal}))$ & 0.61 & 0.23 & 0.65 & 0.21 & 0.68 & 0.21 & 0.71 & 0.20 & 0.76 & 0.19 \\
\hline Niacin $(\mathrm{mg} / 4184 \mathrm{~kJ}(\mathrm{mg} / 1000 \mathrm{kcal}))$ & 11.6 & 1.5 & 11.8 & 1.6 & $12 \cdot 0$ & 1.6 & $12 \cdot 3$ & 1.5 & $12 \cdot 8$ & 1.4 \\
\hline Vitamin $\mathrm{B}_{6}(\mathrm{mg} / 4184 \mathrm{~kJ}(\mathrm{mg} / 1000 \mathrm{kcal}))$ & 0.70 & 0.12 & 0.75 & 0.12 & 0.79 & 0.12 & 0.83 & 0.12 & 0.88 & 0.12 \\
\hline Vitamin $B_{12}(\mathrm{mg} / 4184 \mathrm{~kJ}(\mathrm{mg} / 1000 \mathrm{kcal}))$ & 3.73 & $2 \cdot 27$ & 4.05 & $2 \cdot 30$ & $4 \cdot 37$ & $2 \cdot 22$ & 4.72 & $2 \cdot 18$ & $5 \cdot 30$ & $2 \cdot 10$ \\
\hline Folate $(\mu \mathrm{g} / 4184 \mathrm{~kJ}(\mu \mathrm{g} / 1000 \mathrm{kcal}))$ & 181 & 72 & 202 & 72 & 216 & 71 & 229 & 70 & 245 & 67 \\
\hline Vitamin C (mg/4184 kJ (mg/1000 kcal)) & $55 \cdot 3$ & 23.9 & 67.5 & $24 \cdot 8$ & 73.0 & $24 \cdot 4$ & $78 \cdot 3$ & 23.9 & $84 \cdot 1$ & $22 \cdot 0$ \\
\hline SFA (\% energy) & 6.32 & 2.43 & 6.70 & $2 \cdot 21$ & 6.93 & $2 \cdot 15$ & 7.07 & 1.99 & $7 \cdot 37$ & 1.86 \\
\hline MUFA (\% energy) & 6.32 & $2 \cdot 16$ & 6.81 & 2.02 & $7 \cdot 11$ & 1.95 & 7.36 & 1.85 & 7.78 & 1.76 \\
\hline PUFA (\% energy) & 4.53 & 1.42 & 4.83 & 1.34 & $5 \cdot 11$ & 1.29 & 5.39 & 1.21 & $5 \cdot 69$ & 1.13 \\
\hline$n$-3 PUFA (\% energy) & 0.79 & 0.30 & 0.89 & 0.32 & 0.97 & 0.32 & 1.07 & 0.32 & 1.20 & 0.31 \\
\hline$n-6$ PUFA (\% energy) & 3.73 & 1.18 & 3.93 & $1 \cdot 12$ & $4 \cdot 12$ & 1.08 & 4.31 & 1.00 & 4.47 & 0.91 \\
\hline Cholesterol (mg/4184 kJ (mg/1000 kcal)) & 132 & 61 & 146 & 58 & 155 & 56 & 163 & 53 & 174 & 49 \\
\hline Dietary fibre $(\mathrm{g} / 4184 \mathrm{~kJ}(\mathrm{~g} / 1000 \mathrm{kcal}))$ & 7.34 & 1.61 & 7.93 & 1.57 & 8.34 & 1.58 & 8.78 & 1.58 & $9 \cdot 26$ & 1.52 \\
\hline
\end{tabular}

* Unknown percentage was not shown. 
Table 3. Japanese food scores and all-cause, CVD and cancer mortality (men) (Hazard ratios (HR) and $95 \%$ confidence intervals)

\begin{tabular}{|c|c|c|c|c|c|c|c|c|c|c|c|}
\hline & \multicolumn{2}{|c|}{ Score 0-2 ( $n$ 4694) } & \multicolumn{2}{|c|}{ Score 3 ( $n$ 3502) } & \multicolumn{2}{|c|}{ Score 4 ( $n$ 4238) } & \multicolumn{2}{|c|}{ Score 5 ( $n$ 4752) } & \multicolumn{2}{|c|}{ Score 6-7 (n 5976) } & \multirow[b]{2}{*}{$P_{\text {trend }}$} \\
\hline & $\mathrm{HR}$ & $95 \% \mathrm{Cl}$ & $\mathrm{HR}$ & $95 \% \mathrm{Cl}$ & HR & $95 \% \mathrm{Cl}$ & $\mathrm{HR}$ & $95 \% \mathrm{Cl}$ & $\mathrm{HR}$ & $95 \% \mathrm{Cl}$ & \\
\hline $\begin{array}{l}\text { Person-years } \\
\text { All-cause mortality }\end{array}$ & \multicolumn{2}{|c|}{74938} & \multicolumn{2}{|c|}{56082} & \multicolumn{2}{|c|}{68176} & \multicolumn{2}{|c|}{76767} & \multicolumn{2}{|c|}{97269} & \\
\hline No. of deaths & \multicolumn{2}{|c|}{1186} & \multicolumn{2}{|c|}{925} & \multicolumn{2}{|c|}{1090} & \multicolumn{2}{|c|}{1370} & \multicolumn{2}{|c|}{1738} & \\
\hline Age adjusted & 1.00 & Ref. & 0.94 & $0.86,1.02$ & 0.89 & $0.82,0.96$ & 0.90 & $0.84,0.98$ & 0.86 & $0.80,0.93$ & $<0.001$ \\
\hline Multivariate $^{*}$ & 1.00 & Ref. & 0.96 & $0.88,1.04$ & 0.92 & $0.84,1.00$ & 0.95 & $0.88,1.03$ & 0.93 & $0.86,1.01$ & 0.067 \\
\hline \multicolumn{12}{|l|}{ CVD mortality } \\
\hline No. of deaths & \multicolumn{2}{|c|}{321} & \multicolumn{2}{|r|}{242} & \multicolumn{2}{|c|}{275} & \multicolumn{2}{|r|}{365} & \multicolumn{2}{|c|}{471} & \\
\hline Age adjusted & 1.00 & Ref. & 0.89 & $0.76,1.05$ & 0.82 & $0.69,0.96$ & 0.87 & $0.75,1.01$ & 0.84 & $0.73,0.96$ & 0.011 \\
\hline Multivariate* & 1.00 & Ref. & 0.92 & $0.77,1.08$ & 0.86 & $0.73,1.01$ & 0.90 & $0.77,1.05$ & 0.89 & $0.76,1.04$ & 0.108 \\
\hline \multicolumn{12}{|l|}{ Cancer mortality } \\
\hline No. of deaths & \multicolumn{2}{|c|}{452} & \multicolumn{2}{|c|}{370} & \multicolumn{2}{|c|}{423} & \multicolumn{2}{|r|}{558} & \multicolumn{2}{|c|}{687} & \\
\hline Age adjusted & 1.00 & Ref. & 1.00 & $0.87,1.15$ & 0.92 & $0.81,1.05$ & 1.00 & $0.88,1.13$ & 0.94 & $0.83,1.06$ & 0.272 \\
\hline Multivariate* & 1.00 & Ref. & 1.02 & $0.89,1.17$ & 0.94 & $0.82,1.07$ & 1.05 & $0.93,1.20$ & 1.01 & $0.89,1.14$ & 0.843 \\
\hline
\end{tabular}

Ref., referent values.

* Adjusted for age, geographical region, BMI, education duration, smoking status, alcohol drinking status, sports habits, sleeping duration, history of hypertension and diabetes and total energy intake.

Table 4. Japanese food scores and all-cause, CVD and cancer mortality (women) (Hazard ratios (HR) and $95 \%$ confidence intervals)

\begin{tabular}{|c|c|c|c|c|c|c|c|c|c|c|c|}
\hline & \multicolumn{2}{|c|}{ Score 0-2 ( $n$ 3766) } & \multicolumn{2}{|c|}{ Score 3 ( $n$ 4096) } & \multicolumn{2}{|c|}{ Score 4 ( $n$ 6291) } & \multicolumn{2}{|c|}{ Score 5 ( $n$ 8242) } & \multicolumn{2}{|c|}{ Score $6-7$ ( $n$ 13210) } & \multirow[b]{2}{*}{$P_{\text {trend }}$} \\
\hline & HR & $95 \% \mathrm{Cl}$ & $\mathrm{HR}$ & $95 \% \mathrm{Cl}$ & $\mathrm{HR}$ & $95 \% \mathrm{Cl}$ & $\mathrm{HR}$ & $95 \% \mathrm{Cl}$ & $\mathrm{HR}$ & $95 \% \mathrm{Cl}$ & \\
\hline Person-years & \multirow{2}{*}{\multicolumn{2}{|c|}{61377}} & \multirow{2}{*}{\multicolumn{2}{|c|}{66943}} & \multirow{2}{*}{\multicolumn{2}{|c|}{103740}} & \multirow{2}{*}{\multicolumn{2}{|c|}{138157}} & \multirow{2}{*}{\multicolumn{2}{|c|}{224416}} & \\
\hline All-cause mortality & & & & & & & & & & & \\
\hline No. of deaths & \multicolumn{2}{|c|}{677} & \multicolumn{2}{|c|}{627} & \multicolumn{2}{|c|}{999} & \multicolumn{2}{|c|}{1173} & \multicolumn{2}{|c|}{1907} & \\
\hline Age adjusted & 1.00 & Ref. & 0.89 & $0.80,1.00$ & 0.95 & $0.86,1.05$ & 0.80 & $0.73,0.88$ & 0.76 & $0.70,0.83$ & $<0.001$ \\
\hline Multivariate* & 1.00 & Ref. & 0.92 & $0.82,1.03$ & 0.99 & $0.89,1.09$ & 0.85 & $0.77,0.94$ & 0.82 & $0.75,0.90$ & $<0.001$ \\
\hline \multicolumn{12}{|l|}{ CVD mortality } \\
\hline No. of deaths & \multicolumn{2}{|c|}{250} & \multicolumn{2}{|c|}{201} & \multicolumn{2}{|c|}{327} & \multicolumn{2}{|c|}{387} & \multicolumn{2}{|c|}{569} & \\
\hline Age adjusted & 1.00 & Ref. & 0.79 & $0.66,0.95$ & 0.88 & $0.74,1.03$ & 0.74 & $0.63,0.87$ & 0.64 & $0.55,0.74$ & $<0.001$ \\
\hline Multivariate* & 1.00 & Ref. & 0.80 & $0.67,0.97$ & 0.87 & $0.74,1.03$ & 0.76 & $0.64,0.89$ & 0.66 & $0.56,0.77$ & $<0.001$ \\
\hline \multicolumn{12}{|l|}{ Cancer mortality } \\
\hline No. of deaths & \multicolumn{2}{|c|}{168} & \multicolumn{2}{|c|}{195} & \multicolumn{2}{|c|}{337} & \multicolumn{2}{|c|}{385} & \multicolumn{2}{|c|}{672} & \\
\hline Age adjusted & 1.00 & Ref. & 1.09 & $0.89,1.34$ & 1.23 & $1.02,1.48$ & 1.02 & $0.85,1.23$ & 1.06 & $0.89,1.25$ & 0.790 \\
\hline Multivariate* & 1.00 & Ref. & 1.12 & $0.91,1.38$ & 1.29 & $1.07,1.56$ & 1.09 & $0.90,1.31$ & 1.14 & $0.95,1.36$ & 0.502 \\
\hline
\end{tabular}

Ref., referent values.

* Adjusted for age, geographical region, BMl, education duration, smoking status, alcohol drinking status, sports habits, sleeping duration, history of hypertension and diabetes and total energy intake.

hypertension ${ }^{(27)}$. However, previous studies and our study observed that the score including Japanese pickles intake prevented CVD mortality. $\mathrm{Na}$ intake is positively associated with blood pressure, whereas $\mathrm{K}$ intake is inversely associated ${ }^{(27)}$. Moreover, higher Na:K ratio or high-Na-and-low-K intake was associated with increasing all-cause and/or CVD mortality ${ }^{(28-31)}$. Because vegetables and fruits are renowned for their high $\mathrm{K}$ content, the Na:K ratio reasonably decreases when the Japanese food score increases (Tables 1 and 2). Therefore, a higher Japanese food score representing a larger amount of vegetable and fruit intake may reduce the risk of all-cause and/or CVD mortality.

Participants with higher Japanese food scores had higher mean intakes of almost all nutrients and lower carbohydrate intake in this study. Low carbohydrate and higher protein and fat diets were inversely associated with CVD and all-cause mortality among Japanese women ${ }^{(32)}$. Reviews on the associations between the dietary lifestyle and CVD showed that lower saturated fat and higher $n-3$ polyunsaturated fat intakes might contribute to the lower prevalence and risk of hypercholesterolaemia and CHD among Japanese people, respectively ${ }^{(33)}$. Increased intake of almost all nutrients and decreased intake of carbohydrates due to higher Japanese food score can lead to reduced risk of all-cause and CVD mortality.

This study has several strengths. The present populationbased study relates to its prospective observation design by recruiting a large number of study participants nationwide, which was suitable in calculating the Japanese food scores. As a long-term follow-up study, the outcomes are sufficient to investigate the impact of the Japanese food scores. Furthermore, associated interests were examined by adjusting for potential confounders. However, the following are some limitations of this study. First, the dietary habits of participants were assessed only at the baseline and might have changed 
during the follow-up period; however, this type of data collection methodology is commonly used for epidemiological studies. Second, a large number of participants who had missing data regarding their dietary habits were excluded from the analyses. However, most baseline characteristics (such as BMI, smoking status and alcohol drinking) were similar between the participants and those excluded from the study, except for age $(56.0$ and 56.3 years for study participants $v$. $59 \cdot 2$ and 59.9 years for those excluded from the study and in men and women, respectively) and educational levels of $\geq 13$ years $(17.0 v .8 .4 \%$ in men and $9.8 v .4 .3 \%$ in women, respectively). Therefore, we consider that bias was unlikely in this study, despite the slight differences in baseline characteristics.

In conclusion, adherence to the Japanese food score was associated with decreased risk of all-cause and CVD mortality in a largescale cohort study, especially in women. Our findings can provide evidence that food combinations characterised by the Japanese diet may help prevent mortality in the general population.

\section{Acknowledgements}

The authors sincerely thank Dr Kunio Aoki and Yoshiyuki Ohno, professors' emeritus of the Nagoya University School of Medicine and former chairpersons of the JACC Study, for their encouragement and support during this study. The authors are also greatly indebted to Dr Haruo Sugano, former director of the Cancer Institute, Tokyo, who made substantial contributions to the initiation of the JACC Study; Dr Tomoyuki Kitagawa, director emeritus of the Cancer Institute of the Japanese Foundation for Cancer Research and former project leader of the Grant-in-Aid for Scientific Research on Priority Area 'Cancer'; and Dr Kazuo Tajima, Aichi Cancer Center, who was the previous project leader of the Grant-in-Aid for Scientific Research on Priority Area of Cancer Epidemiology.

This work was supported by the Grants-in-Aid for Scientific Research from the Ministry of Education, Culture, Sports, Science and Technology of Japan (MEXT) (Monbusho); Grants-in-Aid for Scientific Research on Priority Areas of Cancer; and Grants-in-Aid for Scientific Research on Priority Areas of Cancer Epidemiology from MEXT (MonbuKagaku-sho) (nos 61010076, 62010074, 63010074, 1010068, 2151065, 3151064, 4151063, 5151069, 6279102, 11181101, 17015022, 18014011, 20014026 and 20390156).

E. O. analysed the data and wrote the manuscript. A. T., H. I. and $\mathrm{K}$. W. designed and implemented the study. C. D. analysed the FFQ data. K. N., S. U. and all other authors critically revised the manuscript.

The authors declare that there are no conflicts of interest.

\section{References}

1. Alhazmi A, Stojanovski E, McEvoy M, et al. (2014) The association between dietary patterns and type 2 diabetes: a systematic review and meta-analysis of cohort studies. $J$ Hum Nutr Diet 27, 251-260.

2. Bertuccio P, Rosato V, Andreano A, et al. (2013) Dietary patterns and gastric cancer risk: a systematic review and metaanalysis. Ann Oncol 24, 1450-1458.
3. Esposito K, Maiorino MI, Bellastella G, et al. (2015) A journey into a Mediterranean diet and type 2 diabetes: a systematic review with meta-analyses. BMJ Open 5, e008222.

4. Li F, Hou LN, Chen W, et al. (2015) Associations of dietary patterns with the risk of all-cause, CVD and stroke mortality: a meta-analysis of prospective cohort studies. Br J Nutr $\mathbf{1 1 3}$, $16-24$.

5. Schwingshackl L \& Hoffmann G (2014) Adherence to Mediterranean diet and risk of cancer: a systematic review and meta-analysis of observational studies. Int J Cancer 135 , 1884-1897.

6. Siervo M, Lara J, Chowdhury S, et al. (2015) Effects of the Dietary Approach to Stop Hypertension (DASH) diet on cardiovascular risk factors: a systematic review and metaanalysis. Br J Nutr 113, 1-15.

7. Hu FB (2002) Dietary pattern analysis: a new direction in nutritional epidemiology. Curr Opin Lipidol 13, 3-9.

8. Harmon BE, Boushey CJ, Shvetsov YB, et al. (2015) Associations of key diet-quality indexes with mortality in the Multiethnic Cohort: the Dietary Patterns Methods Project. Am J Clin Nutr 101, 587-597.

9. Reedy J, Krebs-Smith SM, Miller PE, et al. (2014) Higher diet quality is associated with decreased risk of all-cause, cardiovascular disease, and cancer mortality among older adults. J Nutr 144, 881-889.

10. Katanoda K, Kim HS \& Matsumura Y (2006) New Quantitative Index for Dietary Diversity (QUANTIDD) and its annual changes in the Japanese. Nutrition 22, 283-287.

11. Nagura J, Iso H, Watanabe $Y$, et al. (2009) Fruit, vegetable and bean intake and mortality from cardiovascular disease among Japanese men and women: the JACC Study. Br J Nutr 102, 285-292.

12. Iso H, Kobayashi M, Ishihara J, et al. (2006) Intake of fish and $n 3$ fatty acids and risk of coronary heart disease among Japanese: the Japan Public Health Center-Based (JPHC) Study Cohort I. Circulation 113, 195-202.

13. Takachi R, Inoue M, Ishihara J, et al. (2008) Fruit and vegetable intake and risk of total cancer and cardiovascular disease: Japan Public Health Center-Based Prospective Study. Am J Epidemiol 167, 59-70.

14. Nakamura Y, Ueshima H, Okamura T, et al. (2009) A Japanese diet and 19-year mortality: national integrated project for prospective observation of non-communicable diseases and its trends in the aged, 1980. Br J Nutr 101, 1696-1705.

15. Tomata Y, Watanabe T, Sugawara Y, et al. (2014) Dietary patterns and incident functional disability in elderly Japanese: the Ohsaki Cohort 2006 study. I Gerontol A Biol Sci Med Sci 69, 843-851.

16. Tamakoshi A, Ozasa K, Fujino Y, et al. (2013) Cohort profile of the Japan Collaborative Cohort Study at final follow-up. J Epidemiol 23, 227-232.

17. Date C, Fukui M, Yamamoto A, et al. (2005) Reproducibility and validity of a self-administered food frequency questionnaire used in the JACC study. J Epidemiol 15, Suppl. 1, S9-S23.

18. Iso H, Date C, Noda H, et al. (2005) Frequency of food intake and estimated nutrient intake among men and women: the JACC Study. J Epidemiol 15, Suppl. 1, S24-S42.

19. Ministry of Education, Culture, Sports, Science and Technology of Japan (2005) Standard tables of food composition in Japan, fifth revised and enlarged edition. Tokyo. http://www. mext.go.jp/b_menu/shingi/gijyutu/gijyutu3/toushin/05031802/ 002.htm (accessed March 2018).

20. Maruyama K, Iso H, Date C, et al. (2013) Dietary patterns and risk of cardiovascular deaths among middle-aged Japanese: JACC Study. Nutr Metab Cardiovasc Dis 23, 519-527. 
21. Mizoue T, Yamaji T, Tabata S, et al. (2005) Dietary patterns and colorectal adenomas in Japanese men: the Self-Defense Forces Health Study. Am J Epidemiol 161, 338-345.

22. Nanri A, Mizoue T, Shimazu T, et al. (2017) Dietary patterns and all-cause, cancer, and cardiovascular disease mortality in Japanese men and women: The Japan public health centerbased prospective study. PLOS ONE 12, e0174848.

23. Shimazu T, Kuriyama S, Hozawa A, et al. (2007) Dietary patterns and cardiovascular disease mortality in Japan: a prospective cohort study. Int J Epidemiol 36, 600-609.

24. Wang X, Ouyang Y, Liu J, et al. (2014) Fruit and vegetable consumption and mortality from all causes, cardiovascular disease, and cancer: systematic review and dose-response meta-analysis of prospective cohort studies. BMI 349, g4490.

25. Zhao LG, Sun JW, Yang Y, et al. (2016) Fish consumption and all-cause mortality: a meta-analysis of cohort studies. Eur J Clin Nutr 70, 155-161.

26. Strazzullo P, D'Elia L, Kandala NB, et al. (2009) Salt intake, stroke, and cardiovascular disease: meta-analysis of prospective studies. BMJ 339, b4567.

27. Dyer AR, Elliott P, Shipley M, et al. (1994) Body mass index and associations of sodium and potassium with blood pressure in INTERSALT. Hypertension 23, 729-736.

28. Judd SE, Aaron KJ, Letter AJ, et al. (2013) High sodium:potassium intake ratio increases the risk for all-cause mortality: the REasons for Geographic And Racial Differences in Stroke (REGARDS) study. J Nutri Sci $\mathbf{2}$, e13.

29. Okayama A, Okuda N, Miura K, et al. (2016) Dietary sodiumto-potassium ratio as a risk factor for stroke, cardiovascular disease and all-cause mortality in Japan: the NIPPON DATA80 cohort study. BMJ Open 6, e011632.

30. Yang Q, Liu T, Kuklina EV, et al. (2011) Sodium and potassium intake and mortality among US adults: prospective data from the Third National Health and Nutrition Examination Survey. Arch Intern Med 171, 1183-1191.

31. Umesawa M, Iso H, Date C, et al. (2008) Relations between dietary sodium and potassium intakes and mortality from cardiovascular disease: the Japan Collaborative Cohort Study for Evaluation of Cancer Risks. Am J Clin Nutr $\mathbf{8 8}$, 195-202.

32. Nakamura Y, Okuda N, Okamura T, et al. (2014) Lowcarbohydrate diets and cardiovascular and total mortality in Japanese: a 29-year follow-up of NIPPON DATA80. Br J Nutr 112, 916-924.
33. Iso H (2011) Lifestyle and cardiovascular disease in Japan. $J$ Atheroscler Thromb 18, 83-88.

\section{Appendix: Members of the Japan Collaborative Cohort Study Group}

The present members of the JACC Study Group who coauthored this paper include Dr Akiko Tamakoshi (present chairperson of the study group), Hokkaido University Graduate School of Medicine; Dr Mitsuru Mori and Fumio Sakauchi, Sapporo Medical University School of Medicine; Dr Yutaka Motohashi, Akita University School of Medicine; Dr Ichiro Tsuji, Tohoku University Graduate School of Medicine; Dr Yosikazu Nakamura, Jichi Medical School; Dr Hiroyasu Iso, Osaka University School of Medicine; Dr Haruo Mikami, Chiba Cancer Center; Dr Michiko Kurosawa, Juntendo University School of Medicine; Dr Yoshiharu Hoshiyama, Yokohama Soei University; Dr Naohito Tanabe, University of Niigata Prefecture; Dr Koji Tamakoshi, Nagoya University Graduate School of Health Science; Dr Kenji Wakai, Nagoya University Graduate School of Medicine; Dr Shinkan Tokudome, National Institute of Health and Nutrition; Dr Koji Suzuki, Fujita Health University School of Health Sciences; Dr Shuji Hashimoto, Fujita Health University School of Medicine; Dr Shogo Kikuchi, Aichi Medical University School of Medicine; Dr Yasuhiko Wada, Faculty of Nutrition, University of Kochi; Dr Takashi Kawamura, Kyoto University Center for Student Health; Dr Yoshiyuki Watanabe, Kyoto Prefectural University of Medicine Graduate School of Medical Science; Dr Kotaro Ozasa, Radiation Effects Research Foundation; Dr Tsuneharu Miki, Kyoto Prefectural University of Medicine Graduate School of Medical Science; Dr Chigusa Date, School of Human Science and Environment, University of Hyogo; Dr Kiyomi Sakata, Iwate Medical University; Dr Yoichi Kurozawa, Tottori University Faculty of Medicine; Dr Takesumi Yoshimura and Yoshihisa Fujino, University of Occupational and Environmental Health; Dr Akira Shibata, Kurume University; Dr Naoyuki Okamoto, Kanagawa Cancer Center; and Dr Hideo Shio, Moriyama Municipal Hospital. 